\title{
The Impact of Sensory Processing Sensitivity (SPS) on Tertiary Learning and Teaching
}

\author{
Kaaryn Cater \\ Poutama Academic Learning Services, Whitireia New Zealand \\ New Zealand
}

\begin{abstract}
Sensory processing sensitivity (SPS) is an innate sensitivity trait that is found in $15-20 \%$ of the population [3] and has been identified in over a hundred species. In the human population, those with this genetic sensitivity are referred to as Highly Sensitive People (HSP) [3]. HSPs tend to process things deeply, and may take longer to process new information [1]. Therefore, in an educational setting, a student could appear to be struggling with new information, or appear to be falling behind with work. Permission was granted to conduct the study 'Does an understanding of HSP help students who identify as HSP to manage their learning?'

A convenience sample of 179 Whitireia students was asked to complete the HSP self-evaluation [2]. 80 students identified as HSP and were invited to take part in a semi-structured interview. From them, 34 students elected to take part in the initial interview and 25 students completed the follow-up interview. Findings show that students rate the knowledge of HSP to be empowering in managing life and study. All students completing the study believe that information on SPS should be provided to all in-coming students, and that tutors should be given training in order to better support HSP students.
\end{abstract}

\section{Introduction}

Sensory processing sensitivity (SPS) is a personality construct that was first identified and described by American psychologist Elaine Aron [3]. It is seen as innate personality trait responsible for processing in-coming internal (pain and hunger) and external (light and noise) sensory stimulation that is found in $15-20 \%$ of the population [2]; [3]; [4]; [6]. The trait has been identified in over a hundred species [18], and the resulting sensitive nervous system is thought to be a genetic sensitivity that may contribute to species survival [3]. In the human population, those with SPS are referred to as Highly Sensitive People (HSP) [2]; [3]. HSPs are more reactive to physical, environmental, emotional and social stimuli and have narrower comfort thresholds than the majority of the population [2]; [3]; [4]. HSPs are also more aware of subtleties in the external and internal environment and tend to process things deeply [3]; [4]; [12]. Zeff [20] refers to 'a highly tuned nervous system' (p.11). Although Aron [3] did not make explicit the role of emotional reactivity in her original findings on HSP, she acknowledges now the fundamental nature of emotional reactivity to the trait [4]. Further, Baumeister, Vohs, DeWall and Zhang [5] believe that emotional reactivity is helpful for memory and learning through providing vital feedback that facilitates introspection, and helps to guide behaviour in new and similar situations.

The HSP measure is the 27 point self-test known as the Highly Sensitive Person Scale (HSPS) which measures responses to general and subtle environmental and internal stimulation [2]; [3]; [4]; [19]. The HSPS was designed by Aron [3] as a result of hundreds of complex interviews and remains in use today, despite substantial scrutiny since its inception in 1997 [19]. Aron [3] developed the scale as a unidimensional construct designed to be independent of emotionality, introversion and gender bias [3] to measure environmental, physical and emotional sensitivities. The HSPS is comprised of face value items such as 'Do you startle easily with loud noise?'; 'Are you overwhelmed by strong sensory input?'; 'Are you easily overwhelmed by bright lights, strong smells, course fabrics or sirens close by?'; 'Are you conscientious?'; 'Are you deeply moved by arts or music?'.

Sobocko and Zelenski [19] highlight that the HSPS has been the subject of rigorous scrutiny and Smolewska, McCabe, and Woody [18] go on to suggest that there is a need for more research in order to inform this relatively new branch of knowledge. Further, Smolewska et al. [18] propose a three factor structure with three separate subgroups rather than a unidimensional construct. They propose the following subgroups: ease of excitation (EOE); 
low sensitivity threshold (LST); and aesthetic sensitivity (AES).

Smolewska et al. [18] conducted studies to explore possible correlations between SPS and Behaviour Inhibition System and Behaviour Activation System (BIS/BAS) and the Big Five with a focus on Neuroticism and Extraversion. It was as a result of this work, that they identified the three subgroups.

The 12 items included in the EOE subgroup show mental overwhelm as a result of external and internal stimulation, for example,"Do you tend to be more sensitive to pain?", "Do you startle easily?". The LST subgroup includes six items that measure unpleasant arousal of external stimuli for example "Are you made uncomfortable by loud noises?", "Do you make a point to avoid violent movies and TV shows?". The AES subgroup includes 7 items measuring aesthetic sensitivities, for example "Are you deeply moved by arts or music?', 'Do you seem to be aware of subtleties in your environment?". Two items were not included in any subgroup as they loaded on more than one component "Are you easily overwhelmed by strong sensory input?" and "Does your nervous system sometimes feel so frazzled that you have to get off by yourself?".

Smolewska et al. [18] conclude that SPS is not associated with neuroticism, as negative emotionality is associated only with the EOE subgroup but not AES and LST, and that this negative emotionality is associated with stress and worry rather than aesthetic appreciation and deep information processing. They found that high BIS correlates relatively highly with EOE, and conclude that most inhibition is associated with reducing excitation. In addition, they [11] found that EOE showed a degree of correlation with neuroticism, although the HSPS relates only moderately to neuroticism. They found a slight correlation between negative extroversion and LST, though found no correlation between negative extraversion and SPS. This supports Aron's findings that SPS is not introversion and that 30 percent of HSPs are extroverts [3]. Openness was the only personality factor found that was associated with HSP, and the authors note that given that HSPs are likely to rate highly for BIS, the tension created between openness and high scoring BIS could lead to overwhelm. They found that AES correlated slightly with openness and suggested that HSPs scoring high on AES and LST could be responsive to incentive and could possibly experience more positive effects when responding to positive stimuli. They conclude that the positive correlations among the factors are consistent with a higher-order construct of SPS.

The study of innate personality traits has been explored under the auspices of a number of disciplines. Pavlov [15] observed fundamentally variant nervous systems and Margaret Mead documented cultural influences on personality traits [14]. Chen, Rubin \& Sun [7] conducted a crosscultural study and found that sensitivity was valued more highly in China than in Canada, and concluded that the valuing of sensitivity is cultural. Kagan [13] refers to Carl Jung's work which identified that there was a minority group who are more influenced by their subconscious than the majority of the population; and noted that this minority group displayed innate sensitivity and intuitiveness. Plomin \& Daniels [16] recognised that people exhibit a wide variation in optimal levels of arousal. Given that HSPs have physical and emotional thresholds that are narrower than the majority of the population; they can therefore be easily overwhelmed [1]. Zeff [20] notes that an HSP's brain waves are often in a theta state and may therefore be more open to subtle stimuli and intuitive feelings. Zeff [20] concludes that this is the state often achieved through deep meditation, and that the deep processing of information associated with this state can lead to feelings of overwhelm if the focus is external rather than internal.

Research into non-human populations has identified similar sensitivity traits - for example the research of Coleman, Clark and Biederman [8] on 'bold' and 'shy' pumpkin seed sunfish found that 'shy' fish ate different food from the bolder fish, inhabited different parts of the pool, and had different parasites on their body. Although the 'shy' fish initially avoided a novel object in the pool (a trap that the bolder fish swam into), it was found that the 'shy' fish adjusted quickly to new situations and ultimately appeared better able to respond to the environment than the bolder fish. Aron [3] went on to suggest a difference in genotype and suggests that the heightened awareness, intuitiveness and perception associated with the sensitivity trait may be a species survival strategy that could indicate a meta-personality trait.

According to Aron \& Aron [3], HSPs tend to process things more deeply than others, and for this reason, may take longer to process new information. In an educational setting, this may present as an apparent lack of understanding of new information, or falling behind with work. This can lead to further levels of overwhelm that can impact on all areas of health and wellbeing. Further, Jaeger [11] asserts that HSPs stay connected with work when it is meaningful, and conversely, are likely to lose interest when work is seen as mundane. Taking longer to assess information has been referred to by Gray [9] in relation to Behaviour Inhibition System (BIS) as 'Stop, look, listen and be ready for action' (p.110). 'Pause to Check' is a term used to describe the 
behaviour of observing and processing before acting [1]; [3]; [4]. It is thought that this taking time to evaluate may allow more elaborate processing of information and lead to deeper learning from experience and applying to future experiences [1]. Baumeister, et al. [5] purport that this is a result of cognitive emotional reactivity.

Acevedo et al. [1] conducted a study using fMRI to observe differences in brain activity between high and low scoring individuals on the HSPS [2]. Although the researchers were looking primarily at participants' reactions to others' emotions, their findings are educationally significant. Acevedo et al. [1] found that the areas of the brain that were active in high HSP scoring individuals were those associated with attention and planning for action: imitation learning; intuition; and understanding other people's goals, motives and intentions.

According to Acevedo et al. [1], knowledge about SPS is still evolving, and there is growing evidence of social advantages of sensitivity. Gregory, Nettelbeck and Wilson [10], conducted a study on openness to experience, intelligence and successful aging and found that there is a link between openness, intelligence and memory. Smolewska et al. [18] also concluded that there are distinct advantages to sensitivity, and drew a link between openness and SPS. Although SPS can lead to some challenges of overwhelm, the positive aspects of sensitivity can be seen as useful for lifelong learning [10], [18].

\section{The Study}

The original motivation for the Whitireia research was based on the hypothesis that there could be issues related to teaching and learning for HSP students in the tertiary setting. Before examining HSPs in the tertiary environment, it was decided to initially establish whether students found it useful to know about SPS and HSP. Ethics approval was granted by the Whitireia Research and Ethics Committee to conduct the study: "Does an understanding of HSP help students who identify as HSP to manage their learning?"

\subsection{Method}

A mixed method approach was deemed the most appropriate for this study in order to build extensive demographic and quantitative data, and to allow for the complexity of qualitative data collected through interviews. The original HSPS responses provided the initial quantitative data. For the purposes of this research, it was decided to use the simplified HSPS [3] in preference to the more complicated research scale which includes Likert ratings for each question. This decision was made as the research question was simply looking to establish whether students found the information useful, and comparative analysis was not planned.

Using the initial HSPS scorings, it was decided to take 12 as the cut-off score, and all students scoring 12 or higher on the HSPS were invited to take part in a semi-structured interview which included four major questions relating to life and study. Students were first asked to rate growing up, school, tertiary study and managing life/study balance on a 6 point Likert scale in order to elicit quantitative values. The interviews progressed according to each student's Likert rating for each question. Extensive qualitative data were collected at this point. At the conclusion of the first interview, students were given an HSP information pack to take away with them. A followup interview was conducted approximately four weeks later where students were asked to rate the usefulness of an awareness of HSP, and the information and resources provided in the information pack.

\subsection{Participants}

A convenience sample of 179 Whitireia students was asked to complete the HSPS self-evaluation [2]. Of these students, 134 elected to complete the questionnaire, and 80 of whom identified as HSP according to the HSPS 12 or higher score threshold. 34 students chose to continue on with the interview. Of these 34 students, 21(62\%) were female and 13 $(38 \%)$ were male. They ranged in age from 18-53 years. 23 participants (68\%) identified as New Zealand Pakeha (New Zealanders of European descent), 3 identified as New Zealand Māori (indigenous people of New Zealand), with the remaining 8 participants of mixed ethnicity (1 English, 1 Chinese, 1 African, and 5 of mixed or unstated ethnicity).

\subsection{Data collection}

Participants who scored 12 or more on the HSPS were invited to take part in a semi-structured interview which included four major questions relating to life and study. Students were first asked to rate growing up, school, tertiary study and managing life/study balance on a 6 point Likert scale in order to elicit quantitative values. The interviews progressed according to each student's Likert rating for each question. Interviews were conducted in a collaborative way, and students were informed that they were under no obligation to answer all questions, and were free to terminate the interview at 
any time. All interviewed students chose to answer all questions. Extensive qualitative data were collected at this point.

At the conclusion of the first interview, students were given an HSP information pack. A follow-up interview was scheduled for several weeks later, at which point students were asked to rate the usefulness of an awareness of HSP, and the information and resources provided.

\subsection{Data Analysis}

Of the 134 students who chose to complete the HSPS, 80 students identified as HSP according to a score of 12 or higher on the HSPS $(60 \%$ of the participating students). The most commonly checked item for the 80 students was in answer to the question "Do other people's mood affect you?"(70 students); the second most commonly checked item was "are you conscientious?"(62 students); with the third most commonly checked item was "Are you annoyed when people try to make you do too many things at once?" (61 students). The fourth most commonly checked item was "Do you get rattled when you have a lot to do in a short amount of time?" (60 students).

Of the 80 who identified as HSP, 34 elected to take part in the initial interview $(43 \%) .25$ students (74\%) completed both interviews, and of those 25 students, $100 \%$ rated the knowledge of HSP to be useful in managing life and study (6 on the 6 point Likhart scale). All students completing the second interview rated the information pack to be moderately helpful (mean score of 4.5 on the 6 point Likert scale).

The volume of qualitative data collected is extensive and can be analysed in a myriad of ways. To date, overall reactions to HSP awareness have been correlated in order to answer the research question. When asked for personal reactions to knowing about HSP, students responded in such ways: "Wow! I feel normal!"; "Knowing about HSP has helped me to deal with things better and build up ways to make me stronger as a person."; "So good to know what's going on!"; "Thank you. I can forgive myself."; "This helps me to deal with on-going stresses!"; "Now I know what I have to do!"; "Thank you. This changes everything."; "Without you I would hang out in the world like a ghost, but thanks to you I am not a ghost anymore".

Of significance, all students who completed the second interview, in answer to the question "What can Whitireia do to help students and tutors to know about HSP?" responded that they thought that the institution should make information about HSP available to all in-coming students and all tutors.

\section{Discussion}

Given that $100 \%$ of respondents at the second interview state that knowledge of HSP is not only useful, but life-changing, and that they think that information about SPS and HSP should be available to students and tutors, there needs to be discussion at the institutional level about how best to provide information and make it available for staff and students. There would need to be buy-in from executive level, and information and training would need to be provided for tutors.

Aron [3] asserts that $15-20 \%$ of the general population is HSP and it would be expected, therefore, that a similar percentage would be identified in the convenience sample used in this study. However, $60 \%$ of the original participants identified as HSP. There could be a number of factors contributing to this higher percentage. The high percentage of people self-identifying as HSP could suggest the threshold of 12 was too low. It was hypothesised that if this were the case, there would be a large differential between the mean scores of the original 80, and of those who chose to follow through with the interview. It was found that the mean score for the initial 80 participants was 17.26 , and the mean score for the 39 interviewed participants was 18.22 . It was concluded that the low threshold may have had a moderate impact on the higher than anticipated number of identifying HSPs.

The majority of students $(78 \%)$ in this study were arts students and this could have increased the percentage of students identifying as HSP. There is a case for further research on actual numbers of HSPs at the institution, with a break-down according to specific programmes.

It is interesting to apply the 3 subgroups to the four most commonly checked items. First, "Do other people's mood affect you?"(87.5\%) (EOE); the second most commonly checked item, "Are you conscientious?"(77.5\%) (AES); the third most commonly checked item, "Are you annoyed when people try to make you do too many things at once?" (76.2\%) (EOE); with the fourth, "Do you get rattled when you have a lot to do in a short amount of time?" $(75 \%)$. Three of the top four items belong to the EOE subgroup, as identified by Smolewska et al. [18]. This is not surprising, given that the 12 items included in EOE measure mental overwhelm as a result of internal or external stimulation. Of significance, it is others' moods that appears most overwhelming for students. This would indicate that a tutor's demeanour and the overall harmony of the classroom plays a significant role in the comfort level of students. The second most commonly checked item 'Are you conscientious?', although 
categorised as AES can be seen as pull between BIS and openness. Students want to do well, but may find it difficult processing new information, which leads to feelings of overwhelm. Finally, it is significant that $76.2 \%$ of students reported that they get annoyed when people try to make them do too many things at once. While it is not surprising that students will tend to become stressed and overwhelmed when faced with multiple concurrent tasks, it is worth noting that this is of particular distress to HSP students.

These findings are useful for exploring how best to support HSP students. In order to do so, institutional and executive buy-in is necessary to drive policy and procedures. In particular, there need to be rigorous guidelines to ensure that all programmes evenly spread work-load throughout the academic year, so as not to overload students and cause overwhelm. In addition, it is imperative for HSP students to receive feedback on their work in a timely manner, as feedback is vital for deep learning in order to apply new knowledge to new situations [5].

It had been hypothesised that the information pack would be of high value to students, but the findings showed it to be of moderate value. It could be further hypothesised from this that by the time HSP students are attending tertiary education, they have already developed useful metacognitive strategies to navigate their sensitivities. This is consistent with the findings of Zeff [20], Smolewska et al. [18], and Gregory, et al [10], all of whom have identified distinct metacognitive advantages to sensitivity.

\section{Limitations}

The major limitation of this study was the initial timing. Students were invited to participate in the $4^{\text {th }}$ term of the academic year. Not only were students busy with end of year exams and assignments, they were reluctant to return to campus once they had completed their studies for the year. Future research would benefit from commencing at the beginning of the year in order to allow time to complete the study.

Self-selection bias may be seen as a limitation of this study, and future research could employ other methods to enlist participants.

The data collection was extremely time consuming, and future studies would benefit from an on-line platform.

Limited ethnic diversity can be seen as a limitation.

\section{Future Research}

There is a paucity of research into the impact of SPS on teaching and learning. Given that all participants in the second interview of this study recommend that information be made available to all in-coming students, there is evidence that further research is needed. In addition, anecdotally, it was noted that many of the students who identified as HSP experienced periods of physical ill-health. Future research could include questions relating to physical health. It has been noted, anecdotally, that HSP students are more likely to seek on-going affirmation of their work from others, and research into this could be useful. There is evidence to suggest that further research is needed into the subgroups and longitudinal studies could seek to establish links between different subgroups and student success through tertiary education and on in the workforce. Given that sensitivity is valued differently culturally, a useful area for research would be some cross-cultural studies comparing the HSP student experience.

\section{References}

[1] Acevedo, B., Aron, E., Aron, A., Sangster, M., Collins, N. and Brown, L. (2014). The highly sensitive brain: an fMRI study of sensory processing sensitivity and response to others' emotions. Brain and Behavior, 4(4), 580-594. doi: $10.1002 / \mathrm{brb} 3.242$

[2] Aron, E. (1996). The highly sensitive person: How to thrive when the world overwhelms you. New York. NY: Harmony Books.

[3] Aron, E., \& Aron, A. (1997). Sensory-processing sensitivity and its relation to introversion and emotionality. Journal of Personality and Social Psychology, 73, 345-368.

[4] Aron, E., Aron, A. \& Jagiellowicz, J. (2012). Sensory processing sensitivity: a review in the light of the evolution of biological resposnivity. Personality and Social Psychology Review, 16(3), 262-282.dx.doi.org/10.1177/ 1088868311434213

[5] Baumeister, F., Vohs, D., DeWall, N. \& Zhang, L. (2007). How emotion shapes behavior: Feedback, anticipation and reflection, rather than direct causation. Personality and Social Psychology Review, 11, 167-203.

[6] Boterberg, S. \& Warreyn, P. (2016). Making sense of it all: The impact of sensory processing sensitivity on daily functioning of children. Personality and individual Differences, 92, 80-86.dx.doi.org/10.1016/ j.paid.2015.12.022.1

[7] Chen, X., Rubin, K. \& Sun, Y. (1992). Social reputation and peer relationships in Chinese and Canadian 
children: A cross-cultural study. Child Development, 63, 1336-1343.

[8] Coleman, K., Clark, A., \& Biederman, L, (1993). Shybold continuum in pumpkinseed sunfish (Lepomis gibbosus): An ecological study of a psychological trait. Journal of Comparative Psychology, 107(3), 250-26.

[9] Gray, J. A. (1991). The neuropsychology of temperament. In J. Strelau \& A. Angleitner (Eds.), Explorations in temperament: International perspectives on theory and measurement (pp.105-128). New York, NY; Plenum Press.

[10] Gregory, T., Nettlebeck, T. \& Wilson, C. (2010). Openness to experience, intelligence and successful aging. Personality and Individual Differences, 48 (8) 895-899. doi.org/10.1016/j.paid.2010.02.017

[11] Jaeger, B. (2004). Making work for the highly sensitive person. New York, NY: McGraw-Hill Books.

[12] Jagiellowicz, J. A. (2012). The relationship between the temperament trait of sensory processing sensitivity and emotional reactivity. A thesis submitted to Stony Brook University in partial fulfilment of the requirements for the degree of Doctor in philosophy in social/health psychology. Unpublished thesis, Stony Brook University, New York.

[13] Kagan, J. (1994). Galen's prophecy: Temperament in human nature. New York, NY: Basic Books.

[14] Mead, M. (1963). Sex and temperament in three primitive societies. New York, NY: Morrow.

[15] Pavlov, I. (1927). Conditioned reflexes. London, UK: Oxford University Press.

[16] Plomin, R. \& Daniels, D. (1986). Genetics and shyness. In W. H. Jones, J. M. Cheek, \& S. R. Briggs (Eds.). Shyness: Perspectives on research and treatment. New York, NY: Plenum.

[17] Sih, A., Bell, A., \& Johnson, J.C. (2004). Behavioral syndromes: An integrative overview. Quarterly Review of Biology, 79, 241-277.

[18] Smolewska, K., McCabe, S. \& Woody, E. (2006). A psychometric evaluation of the Highly Sensitive People Scale: The components of sensory-processing sensitivity and their relation to BIS/BAS and "Big Five". Personality and Individual Differences, 40, 1269-1279.

Dx.doi.org/10.1016/j.paid.2005.09.022
[19] Sobocko, K. \& Zelenski, J. (2015). Trait sensoryprocessing sensitivity and subjective well-being: Distinctive associations for different aspects of sensitivity. Personality and Individual Difference, 83, 44-49. dx.doi.org/10.1016/j.paid.2015.03.045

[20] Zeff, T. (2004). The highly sensitive person's survival guide. Oakland. CA: New Harbinger Publications. 
International Journal of Technology and Inclusive Education (IJTIE), Volume 6, Issue 1, June 2017 